\title{
Demência com corpos de Lewy: uma revisão para o psiquiatra
}

\author{
Almir Tavares ${ }^{1}$ \\ Camilo Azeredo ${ }^{1}$
}

Recebido: 22/9/2002 Aceito: 18/12/2002

\section{RESUMO}

A demência com corpos de Lewy (DCL) tornou-se hoje uma entidade nosológica distinta, considerada por muitos autores a segunda causa principal de demência degenerativa, atrás apenas da doença de Alzheimer. Sua neuropatologia é caracterizada pela presença de corpos de Lewy tanto em regiões corticais como em áreas subcorticais do encéfalo. As principais manifestações clínicas incluem: declínio cognitivo progressivo, alucinações visuais recorrentes, flutuação no estado cognitivo e sinais parkinsonianos. Observa-se maior sensibilidade a efeitos indesejáveis de medicamentos neurolépticos e a resposta a medicações inibidoras da acetilcolinesterase é geralmente boa.

Unitermos: Demência com corpos de Lewy; Doença de Alzheimer; Neurolépticos; Alucinações.

\section{ABSTRACT}

\section{Dementia with Lewy bodies: a review for psychiatrists}

Lewy body dementia became today a distinct nosological entity, considered by many authors the second cause of degenerative dementia, after Alzheimer's disease. Its neuropathology is caracterized by the presence of Lewy bodies both in cortical and subcortical areas of the brain. The main clinical manifestations are: progressive cognitive impairment, recurrent visual hallucinations, fluctuating cognitive status and parkinsonian signs. There is greater sensitivity to side effects of neuroleptic drugs and the response to cholinesterase inhibitors is generally good.

Keywords: Dementia with Lewy bodies; Alzheimer's disease; Neuroleptics; Hallucinations.

\section{Introdução}

Demência é uma síndrome clínica caracterizada por déficits cognitivos múltiplos, adquiridos e persistentes, capazes de interferir de maneira substancial nas atividades de vida diária do paciente (Tavares, 1992; Cummings e Reichman, 1998). É mais prevalente nos segmentos da população com idade mais avançada, principalmente naqueles com mais de 75 anos (Cummings e Reichman, 1998). A doença de Alzheimer (DA) e a demência com corpos de Lewy (DCL) são os principais representantes de demências neurodegenerativas.
Caracterizada por um excessivo número de placas senis e de emaranhados neurofibrilares no exame histopatológico do encéfalo, a doença de Alzheimer tem início insidioso e gradual, havendo um declínio progressivo, lento e simultâneo da memória, do aprendizado, da linguagem e das habilidades visoespaciais (Diniz e Tavares, 2001; Tavares, 1985). A demência com corpos de Lewy se apresenta com declínio cognitivo, alucinações visuais recorrentes, flutuação no estado cognitivo, sinais parkinsonianos extrapiramidais, sensibilidade aumentada ao uso de neurolépticos. Ao exame neuropatológico, presença de corpos de Lewy em regiões corticais e subcorticais.

Projeto Lar dos Idosos (www.medicina.ufmg.br/laridoso) da Universidade Federal de Minas Gerais e Departamento de Psiquiatria e Neurologia da Faculdade de Medicina da UFMG.

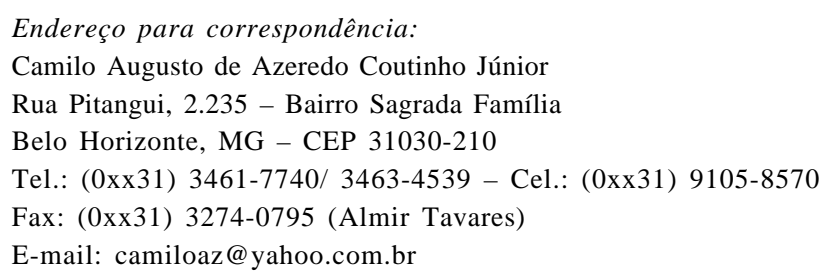


A expressão "demência com corpos de Lewy" foi proposta recentemente, com a finalidade de simplificar e de unificar um grande número de terminologias empregadas por diferentes autores para se referir ao mesmo quadro clínico (variante com corpos de Lewy da doença de Alzheimer, demência associada com corpos de Lewy corticais, demência senil do tipo corpos de Lewy, doença com corpos de Lewy difusos e doença de Alzheimer com variações parkinsonianas). A partir daí, a DCL vem ganhando visibilidade como uma verdadeira entidade nosológica distinta (McKeith et al., 1996).

Sendo a DCL uma demência do tipo Alzheimer, alguns autores ainda não aceitam distingui-la da doença de Alzheimer, preferindo considerá-la uma variante desta última. Quando considerada separadamente da doença de Alzheimer, a demência com corpos de Lewy torna-se a segunda demência neurodegenerativa mais prevalente (Papka, Rubio e Schiffer, 1998; Tomlinson, 1970). Kosaka et al. (1984) observaram que cerca de $15 \%$ a $25 \%$ dos cérebros autopsiados de pacientes idosos demenciados possuíam os achados histopatológicos (corpos de Lewy) fundamentais para o diagnóstico de DCL, no tronco cerebral e no córtex.

Num estudo retrospectivo de 19 casos de DCL confirmados por exame neuropatológico, Maeda et al. (2000) observaram que a idade de início da doença variou de 50 a 88 anos (média de 75,9 anos) e que sua duração até o óbito variou de 1 a 14 anos.

Diagnosticar DCL provável é particularmente relevante em dois aspectos: em primeiro lugar, em virtude dos cuidados necessários com relação ao risco advindo do uso de medicações neurolépticas; e, em segundo lugar, por conta do significativo benefício para estes pacientes advindo do tratamento com drogas inibidoras da acetilcolinesterase.

\section{Etiopatogênese e achados neuropatológicos}

Os corpos de Lewy (CL) (Lewy, 1912; 1913) são os achados histológicos fundamentais para o diagnóstico patológico da DCL. Estruturas de localização intracitoplasmática são esféricos e eosinofílicos. Podem ser identificados pela coloração com a hematoxilina-eosina ou por meio de método imunocitoquímico, com antiubiquitina. Também podem localizar-se fora de neurônios. Podem apresentar outras formas, como a ovóide e as alongadas. Do ponto de vista histológico, podem ser classificados em CL clássicos e CL corticais. O termo CL clássicos é aplicado para as estruturas com o centro hialino circundado por um halo claro, normalmente observadas em neurônios da substância negra e do locus ceruleus. Os $C L$ corticais são estruturas bem menos definidas, sem halo claro e encontradas em neurônios corticais. Embora menos freqüentes, os neuritos de Lewy (NL) possuem uma significância semelhante aos CL para o diagnóstico patológico da DCL e são formados por prolongamentos neuronais dilatados, contendo inclusões fusiformes ou ovóides. O desenvolvimento de novos métodos para detecção de corpos de Lewy e de neuritos de Lewy será fundamental para os avanços nos estudos anatomopatológicos da DCL.

As áreas de predileção dos CL são: tronco encefálico, núcleos subcorticais, córtex límbico (cíngulo, entorrinal e amígdala) e o neocórtex (temporal > frontal = parietal). Os NL são encontrados principalmente no hipocampo (CA 2-3), amígdala, núcleo basal de Meynert e núcleo dorsal do vago.

Dependendo das suas localizações no cérebro, diferentes condições clínicas se associam com a presença dos CL; corpos de Lewy situados na via nigroestriatal estarão ligados a manifestações extrapiramidais; nos gânglios autonômicos, à hipotensão postural; no córtex límbico, à psicose e, no neocórtex, com declínio cognitivo (Crystal, 2002).

Em alguns encéfalos de pacientes com DCL pode haver a coexistência dos corpos de Lewy com as placas senis e os emaranhados neurofibrilares, estruturas presentes em abundância na DA e em menor quantidade em encéfalos de pacientes idosos sem demência. Porém, o achado de CL não é exclusivo da DCL: no envelhecimento normal do encéfalo e na doença de Alzheimer podemos observá-los, em menor quantidade. Também são encontrados no encéfalo na demência com corpos de Lewy: perda regional de neurônios; microvacuolização (mudança espongiforme); perda de sinapses; anormalidades neuroquímicas; e déficits de neurotransmissores (Crystal, 2002).

O diagnóstico post-mortem de DCL dependerá da quantidade e da localização dos CL, sendo o tronco encefálico e o córtex cerebral as localizações fundamentais para o diagnóstico dessa demência (McKeith, 1996).

Os CL são formados principalmente por proteínas $\alpha$-sinucleína, por proteínas neurofilamentares e pela ubiquitina. Suspeita-se que a $\alpha$-sinucleína pré-sináptica seja a principal envolvida na formação desses corpúsculos, sendo fatores genéticos e/ou epigenéticos os possíveis responsáveis pelo surgimento dos CL nos neurônios (Sung et al., 2001; Trojanowaski e Lee, 1998). Os CL, além de representarem neurônios que sofreram degeneração, possivelmente exercem efeito neurotóxico regional. 
Em condições normais, aparentemente, a $\alpha$-sinucleína interage com uma grande variedade de fosfolípides de membrana, participa na regulação da diferenciação celular, na plasticidade sináptica, na sobrevivência celular e na neurotransmissão dopaminérgica (Lucking, 2000).

Em alguns grupos familiares portadores de doença de Parkinson familial, pôde-se observar uma importante mutação no gene da $\alpha$-sinucleína. Esta proteína atípica possui uma substituição de uma alanina e uma treonina na posição 53 (A53T). Uma hipótese proposta para explicar o surgimento dos CL seria que essa alteração molecular predisporia a $\alpha$-sinucleína a agregar-se entre outras proteínas da mesma classe ou entre proteínas de outras classes (por exemplo: subunidades neurofibrilares) (Polymeropoulos et al., 1997). No entanto, em estudo recente, Higuchi et al. (1998) avaliaram a presença dessa mutação (A53T) em 329 casos esporádicos de DP, DCL e DA e sua presença não foi observada em nenhum dos casos, o que sugere o não envolvimento dessa mutação nessas doenças neurológicas.

Os radicais livres também podem estar associados a agregações anômalas entre proteínas citoplasmáticas, causando então a formação dos CL e o conseqüente processo de neurodegeneração. Podem ainda estar envolvidos em mutações do gene da $\alpha$-sinucleína (Ostrerova-Golts et al., 2000). Disfunções no DNA mitocondrial, aumento dos níveis de ferro livre e a perda ou diminuição dos mecanismos de defesa contra radicais livres são outras possíveis fontes indutoras do aumento do estresse oxidativo no paciente com doença de Parkinson e DCL (Foley e Riederer, 2000). A ativação aumentada da enzima heme-oxigenase-1 (HO-1), que normalmente é responsável pela degradação do grupo heme em biliverdina, do ferro livre e de monóxido de carbono, pode estar associada com a DCL. Aventa-se a possibilidade de que essa enzima seria responsável pela deposição aumentada de ferro e conseqüente dano mitocondrial, exercendo um efeito neurodegenerativo (Schipper, 2000).

Danos oxidativos em ácidos nucléicos podem contribuir de maneira significativa com a patogênese da neurodegeneração dopaminérgica da substância negra de pacientes com doença de Parkinson (DP) e nos portadores de DCL. Por meio de estudos imunoistoquímicos, pôde-se identificar um importante produto do estresse oxidativo de ácidos nucléicos, a hidroxiguanosina (8-HGO). Em pacientes com DP, a imunorreatividade da 8-HGO citoplasmática na substância negra está aumentada, e discretamente elevada nos núcleos dorsal da rafe e oculomotor. Nos pacientes com DCL observa-se um aumento importante da 8-HGO citoplasmática, porém não tão proeminente quanto nos porta- dores de DP. Um dado bastante importante é que a imunorreatividade da 8-HGO nuclear não foi observada nesses pacientes, mostrando que o dano oxidativo é mais restrito aos ácidos nucléicos do citoplasma, sendo o RNA e o DNA mitocondrial os principais alvos do estresse oxidativo (Zhang et al., 1999).

Um excesso de compostos carbonil reativos e seus produtos de reação (produtos finais de glicalização avançada - PFGA) podem estar envolvidos na patogênese de desordens neurodegenerativas, e em particular naquelas associadas ao acúmulo de CL (DP e DCL). Nelas o acúmulo de PFGAs estaria envolvido intimamente com a agregação anormal de proteínas neuronais e com a subsequiente formação de CL (Munch et al., 2000).

O verdadeiro papel do sistema imunológico na patogênese da demência com corpos de Lewy ainda não é bem conhecido. Sabe-se que os cérebros dos pacientes com DCL apresentam um processo inflamatório significativo. Por meio de estudos neuropatológicos comparativos, concluiu-se que cérebros de indivíduos com DCL apresentavam uma quantidade bem maior de células da micróglia ativadas do que indivíduos não demenciados. Esse estado inflamatório crônico pode ser o mecanismo envolvido na perda da função ou da degeneração dos neurônios. As células da micróglia ativadas secretam mediadores pró-inflamatórios com alto caráter neurotóxico, por isso podem estar relacionadas com a gênese e com a progressão da DCL. Outro dado importante é que a quantidade de células da micróglia é proporcional ao número de $\mathrm{CL}$ em uma determinada região (Ian e Mackenzie, 2000; Togo, 2001).

Existem evidências de que a atividade colinérgica no neocórtex na demência com corpos de Lewy é menor do que na doença de Alzheimer, havendo, principalmente, um envolvimento das enzimas acetilcolinetransferase $\mathrm{e}$ acetilcolinesterase. A diminuição da neurotransmissão monoaminérgica no estriado também pode ser observada nos pacientes com DCL. Anormalidades dos receptores dopaminérgicos na substância negra são possíveis responsáveis pela sensibilidade aumentada ao uso de neurolépticos.

Uma hipótese bastante interessante correlaciona o possível papel do vírus Epstein-Barr (EBV) na formação dos corpos de Lewy. Anticorpos gerados contra a proteína 1 da membrana do EBV mostram uma grande imunorreatividade aos CL (mais especificamente à $\alpha$-sinucleína) e às inclusões citoplasmáticas da glia. Essa reação cruzada entre antígenos de membrana do EBV e a $\alpha$-sinucleína pode implicar em possíveis mecanismos infecciosos ou auto-imunes na patogenia da DCL (Woulfe et al., 2000). 


\section{Aspectos clínicos da DCL}

Tomando-se os critérios propostos pelo Consenso sobre demência com corpos de Lewy, podemos chegar a um diagnóstico de DCL provável ou de DCL possível, sendo o diagnóstico definitivo dependente de estudo anatomopatológico post-mortem (Tabela 1).

A presença de um declínio progressivo das funções cognitivas do paciente que interfira nas funções de vida diária é uma exigência fundamental para o diagnóstico da demência com corpos de Lewy.

Acometimento importante da memória pode não ocorrer nos estágios precoces da DCL, porém se torna evidente com a progressão dessa demência. No paciente com DCL nota-se uma deterioração da capacidade de evocação da memória, sendo os declínios relacionados à aquisição e à consolidação da memória mais evidentes nos pacientes com DA. Podem ser particularmente proeminentes os déficits nos testes de atenção e nas capacidades visuoespaciais. Em pacientes demenciados com escore menor ou igual a 13 no Mini-Mental State Examination de Folstein (Folstein e McHugh, 1975), uma incapacidade de se reproduzir os pentágonos é mais sugestiva de DCL que de DA (Ala et al., 2001)

Flutuações nas funções cognitivas são comuns na DCL. Nos estágios precoces, os pacientes podem mostrar déficits da função cognitiva e da performance global, alternando com períodos de performance normal ou próximo do normal. A periodicidade e a amplitude da flutuação pode variar entre indivíduos, não havendo um padrão comum a todos os pacientes com DCL. A flutuação pode ser rápida (minutos ou horas) ou em períodos maiores de tempo (semanas ou meses). A constatação da flutuação se dá através de informações do próprio paciente, dos cuidadores ou do acompanhamento pelo médico. Flutuações do estado cognitivo ao entardecer do dia (sundowning) pode ser possível achado na DCL, porém pode ser observado também em demências de outras etiologias (McKeith et al., 1996).

Alucinações visuais bem formadas, detalhadas e com caráter recorrente foram relatadas na maioria dos grupos com DCL investigados. Alucinações auditivas também podem ser encontradas, sendo, porém, menos comuns. Os pacientes com alucinações podem responder a estas de diversas maneiras; essa resposta depende principalmente de seu insight e da sensibilidade dos cuidadores em seu manejo. Em recente estudo, as alucinações visuais mostraram-se mais freqüentes num grupo de pacientes com demência com corpos de Lewy do que num grupo com doença de Alzheimer, não havendo entretanto diferenças significativas entre os grupos de pacientes com doença de Parkinson e DCL (Lisa et al., 1996). O manejo desse tipo de manifestação é difícil, pois, normalmente, os pacientes com DCL apresentam uma sensibilidade especialmente aumentada aos efeitos indesejáveis dos neurolépticos, tornando o seu controle bastante complexo.

Sinais extrapiramidais parkinsonianos também podem ser observados no paciente com DCL. Rigidez e bradicinesia são os achados mais comumente observados. Fácies em máscara, fala arrastada e hipofonética, tremor e marcha lentificada e arrastada são encontrados nesses pacientes. Nos indivíduos mais idosos com DCL, o tremor em repouso é menos comum. A ordem de início dos quadros mental e motor é variável e de difícil definição nos pacientes mais velhos, pois, freqüentemente, apresentam um início quase que simultâneo dos sintomas mentais e extrapiramidais. Quando a demência ocorrer dentro dos 12 meses de início dos sinais e sintomas motores extrapiramidais, o paciente deverá receber o diagnóstico primário de DCL possível, que será fortalecido pela presença de um aspecto essencial ou de um sintoma de suporte de acordo com os critérios de diagnóstico clínico da DCL (Tabela 1). Caso a história clínica de parkinsonismo tenha início mais de 12 meses depois do início do declínio cognitivo, o diagnóstico mais apropriado a ser usado será doença de Parkinson com demência, DCL ou outro (McKeith et al., 1996).

Em estágios avançados da doença de Alzheimer e de outras demências, pode haver sinais parkinsonianos e, em fases terminais, pode haver paraplegia em flexão. A apresentação precoce do parkinsonismo no curso de uma demência é um achado consistente para o diagnóstico de DCL, porém não é específico para ela. Neurolépticos, mesmo em baixas doses, podem induzir parkinsonismo em idosos ou em pacientes demenciados. A DCL pode ser diferenciada do parkinsonismo induzido por neuroléptico pela persistência dos sintomas motores extrapiramidais após a retirada da droga.

O uso de medicamentos neurolépticos pode piorar ou ainda precipitar as manifestações extrapiramidais nos pacientes com DCL, aumentando significativamente a morbidade e a mortalidade desse enfermo. Aproximadamente $81 \%$ dos idosos com DCL vão apresentar algum tipo de efeito adverso, e mais da metade dos indivíduos sensíveis aos neurolépticos apresentarão reações severas. Sedação, aumento da confusão, rigidez e imobilidade são algumas reações graves observadas. Em outros casos podemos observar o aparecimento da síndrome neuroléptica maligna, com febre, rigidez generalizada e aumento dos níveis séricos da CPK. Cabe notar que cerca de $7 \%$ dos pacientes com DA apresentam efeitos adversos com o uso de antipsicóticos (McKeith et al., 1992).

Tendência a quedas repetidas, síncopes e perda transitória da consciência são achados de suporte para 
Tabela 1 Critérios para diagnóstico clínico de demência com corpos de Lewy possível e provável

1. O sinal cardinal da DCL é o declínio cognitivo de magnitude suficiente para interferir na função social e profissional do doente. Uma perda proeminente e persistente da memória não ocorre necessariamente nas fases iniciais da doença, embora se torne evidente com a evolução. Defeitos da atenção, das perícias subcorticais e da capacidade visoespacial são evidentes.

2. Dois dos seguintes aspectos são necessários para o diagnóstico de doença provável e um para o diagnóstico de doença possível:

a) flutuações das capacidades cognitivas, com variações pronunciadas da atenção e da vigilância;

b) alucinações visuais recorrentes que são tipicamente bem formadas e detalhadas;

c) parkinsonismo espontâneo.

3. Características que apóiam o diagnóstico:

a) quedas repetidas;

b) síncope;

c) perdas transitórias de consciência;

d) sensibilidade aos neurolépticos;

e) ilusões sistematizadas;

f) alucinações em outras modalidades.

4. O diagnóstico de DCL é menos comum na presença de:

a) doença isquêmica evidente através de sinais neurológicos focais ou por evidências em neuroimagem;

b) evidência no exame físico de outra doença sistêmica ou neurológica que justifique o quadro.

Adaptado de: "Consensus guidelines for the clinical and pathological diagnosis of dementia with Lewy bodies", 1996

o diagnóstico da DCL. Nesse caso, os ataques de síncopes com perda completa da consciência e do tônus muscular podem estar associados com a presença de CL no tronco cerebral e no sistema nervoso autonômico. No entanto, demências de qualquer etiologia são fatores de risco para esses três achados clínicos, bem como, sinais e sintomas neurológicos focais associados a esses quadros podem também ser indicativos de ataque isquêmico transitório (McKeith et al., 1996).

Um fato fundamental na avaliação de qualquer idoso acometido por demência é a identificação de doenças sistêmicas, cerebrovasculares ou de outras desordens cerebrais que possam explicar seu surgimento. No entanto, a presença de qualquer um desses elementos não irá exclui por completo o diagnóstico de DCL, uma vez que esses tipos de comorbidades em pacientes idosos não são incomuns. Deve-se suspeitar ou excluir uma demência de origem vascular por meio de critérios clínicos e radiológicos. $\mathrm{O}$ aparecimento de mioclonia em pacientes com uma forma rapidamente progressiva de DCL pode levar a uma suspeita clínica de doença de CreutzfeldtJakob. Em pacientes com delirium intermitente, exames clínico e laboratorial apropriados durante a fase aguda maximizam a chance de se detectar fatores infecciosos, metabólicos, inflamatórios e de outras etiologias.

Uma causa comum de declínio das habilidades intelectuais nos idosos é a pseudodemência, também conhecida como síndrome demencial da depressão. É caracterizada por esquecimento, retardo psicomotor, desmotivação e lentidão cognitiva. Os pacientes apresentam evidências de humor deprimido e história familiar ou pessoal de depressão. O uso de antidepressivos leva à regressão do quadro neuropsiquiátrico.
Os pacientes com esquizofrenia podem exibir uma grande variedade de anormalidades cognitivas, mas essas condições apresentam início precoce e está associada a uma grande variedade de sintomas distintos (Cummings e Reichman, 1998).

Os pacientes com DCL apresentam boa resposta ao uso dos inibidores da acetilcolinesterase. Em muitos casos, apresentam melhor resposta ao tratamento com esses medicamentos do que pacientes portadores de doença de Alzheimer. A dose de rivastigmina a ser empregada na DCL encontra-se entre $6 \mathrm{mg}$ a $12 \mathrm{mg}$ por dia (McKeith et al., 2000; Wesnes et al., 2000). Recentemente, a eficácia terapêutica do donepezil em pacientes com DCL tem sido anunciada, sendo os benefícios tanto cognitivos quanto relacionados às manifestações extrapiramidais e comportamentais. As doses de donepezil preconizadas variam de $5 \mathrm{mg}$ a 10 mg por dia (Arahata et al., 2001; Rojas-Fernandes, 2001; Skjerve e Nygaard, 2000). O uso da tacrina mostra-se menos promissor comparado aos outros inibidores da acetilcolinesterase (Querfurth et al., 2000).

\section{Conclusões}

Estudos mais detalhados sobre a doença com corpos de Lewy são essenciais para sua melhor compreensão, para maior divulgação do conceito e para a sua aceitação como uma demência que apresenta peculiaridades distintas da doença de Alzheimer.

O conhecimento dessa entidade nosológica é de fundamental importância para o psiquiatra, visto o maior risco do surgimento nesses pacientes da síndrome neuroléptica maligna. 


\section{Referências bibliográficas}

Ala, T.A.; Hughes, L.F.; Kyrouac, G.A.; Ghobrial, M.W.; Elble R.J. Pentagon Copying is More Impaired in Dementia with Lewy Bodies than in Alzheimer's Disease. J Neurol Neurosurg Psychiatry 70(4): 483-8, 2001.

Arahata, H.; Ohyagi, Y.; Matsumoto, S. et al. - A Patient with Probable Dementia with Lewy Bodies, Who Showed Improvement of Dementia and Parkinsonism by the Administratim of Donepezil. Rinsho Shinkeigaku 41(7): 402-6, 2001.

Crystal, H.A. - Dementia with Lewy Bodies. Disponível em: www.emedicine.com/neuro/topic91.htm. Acesso em 3 de janeiro de 2002.

Cummings, J.L.; Reichman W.E. - Dementia. In: Duthie, EHJ.; Katz, PR. Practice of Geriatrics. $3^{\text {rd }}$ ed. WB Saunders Company. Philadelphia, pp. 268-78, 1998.

Dickson, D.W.; Ruan, D.; Crystal, H. et al. - Hippocampal Degeneration Differentiates Diffuse Lewy Body Disease (DLBD) From Alzheimer's Disease: Light and Electron Microscopic Immunocytochemistry of CA2-3 Neuritis Specific to DLBD. Neurology 41: 1402-9, 1991.

Diniz, B.S.O.; Tavares, A. - As Bases Biológicas da Doença de Alzheimer. Rev Med Minas Gerais 11(2): 92-7, 2001.

Foley, P.; Riederer, P. - Influence of Neurotoxins and Oxidative Stress on Onset and Progression of Parkinson's disease. J Neurol 247 (Suppl. 2): II82-94, 2000.

Folstein, MF.; Folstein, SE.; McHugh. - Mini Mental State: A practical Method of Grading Cognitive State of Patients for the Clinician. J Psychiatr Res 12: 189-98, 1975.

Higuchi, S.; Arai H.; Matsushita, S.; Matsui, T.; Kimpara, T.; Takeda, A.; Shirakura, K. Mutation in the alpha-synuclein gene and sporadic parkinson's disease, Alzheimer's disease and dementria with leroy bodies. Exp Neurol 153(1): 164-6, 1998.

Ian, RA.; MacKenzie, M.D. - Activated Microglia in Dementia With Lewy Bodies. Neurology 55: 132-4, 2000.

Kosaka, K.; Yoshimura, M.; Ikeda, K. et al. - Diffuse Type of Lewy Body Disease: Progressive Dementia with Abundant Cortical Lewy Bodies and Senile Changes of Varying Degree - A New Disease? Clin Neuropathol 3: 185-92, 1984.

Lewy, F.H. - Paralysis Agitans. I. Pathologische Anatomie. In: Handbuch Der Neurologie. ed. Lewandowsky M, Springer, vol 3, Berlin, pp. 920-33, 1912.

Lewy F.H. - Zur Pathologishen Anatomie der Paralysis Agitans. Deut Zeit Nervenheilk 50: 50-5, 1913.

Lisa, AK.; Elan, D.L.; RandolPh, B.S. - Psychiatric Features in Diffuse Lewy Body Disease: A Clinicopathologic Study Using Alzheimer's Disease and Parkinson's Disease Comparison Groups. Neurology 47: 1148-52, 1996.

Lucking, C.B.; Brice, A. - Alpha-Synuclein and Parkinson's Disease. Cell Mol Life Sci 57 (13-14): 1894-908, 2000.

Maeda, A.; Yamada, M.; Yoshida, R.; Itoh, Y.; Sugai, Y.; Suematsu, N.; Отомо, E.; Mizusawa, H. - Clinical Features of Late Onset Dementia With Lewy Bodies. Insho Shinkeigaku 40(10): 98692, 2000.

Martin-Ruiz, C.; Court, J.; Lee, M.; Piggott, M.; Johnson, M.; B allard, C.; Kalaria, R.; Perry, R.; Perry, E. - Nicotinic Receptors in Dementia of Alzheimer, Lewy Body and Vascular Types. Acta Neurol Scand Suppl 176: 34-41, 2000.

McKeith, I.G.; Galasko, D.; Kosaka, K.; Perry, E.K.; Dickson, D.W.; Hansen, L.A.; Salmon, D.P.; Lowe, J.; Mirra, S.S.; Byrne, E.J.; Lennox, G., Quinn, N.P.; Edwardson, J.A.; Ince, P.G.; Bergeron, C.; Burns, A.; Miller, B.L.; Lovestone, S.; Collerton, D.; Jansen E.N.H.; Ballard, C.; De Vos, R.A.I.; Wilcock, GK.; Jellinger,
K.A.; Perry R.H. - Consensus Guidelines for the Clinical and Pathological Diagnosis of Dementia with Lewy Bodies (DLB): Report of The Consortium on DLB International Workshop. Neurology 47: 1113-24, 1996.

McKeith, I.G.; Fairbain, A.; Perry, R.; Thompson, P.; Perry, E. Neuroleptic Sensitivity in Patients with Senile Dementia of Lewy Body Type. BMJ 305: 673-8, 1992.

Munch, G.; Luth, H.J.; Wong, A.; ARendT, T.; HIRSCH, E.; RAVID, R.; Riederer, P. - Crosslinking of Alpha-Synuclein by Advanced Glycation Endproducts - An Early Pathophysiological Step in Lewy Bodies Formation? J Chem Neuroanat 20(3-4): 253-7, 2000.

Ostrerova-Golts, N.; Petrucelli, L.; Hardy, J.; Lee, J.M.; Farer, M.; Wolozin, B. - The A53T alpha-synuclein mutation increases iron-dependent aggregation and toxicity. J Neurosci Sci 20(16): 6048-54, 2000.

PAPKA, M.; Rubio, A.; Schiffer R.B. - A Review of Lewy Body Disease, an Emerging Concept of Cortical Dementia. J Neuropsychiatry Clin Neurosci 10: 267-79, 1998.

Polymeropoulos, M.H.; Laedan, C.; Leroy, E. et al. - Synuclein gene identified in families with Parkinson's disease. Science 274: 1197-9, 1997.

Querfurth, H.W.; Allam, G.J.; Gefrroy, M.A.; Schiff, H.B.; Kaplan, R.F. - Acetylcholinesterase Inhibition in Dementia with Lewy Bodies: Results of a Prospective Pilot Trial. Dement Geriatr Cogn Disord 11(6): 314-21, 2000.

Rojas-Fernandes, C.H. - Successful Use of Donepezil for the Treatment of Dementia with Lewy Bodies. Ann Pharmacother 35(2): 202-5, 2001

SkJerve, A.; NygaArd, H.A. - Improvement in Sundowning in Dementia with Lewy Bodies After Treatment with Donepezil. Int J Geriatr Psychiatry 15(12): 1147-51, 2000.

SCHipPeR, HM. - Heme Oxygenaase-1: Role in Brain Aging and Neurodegeneration. Exp Gerontol 35(6-7): 821-30, 2000.

Sung, J.Y.; Kim, J.; PAIK, S.R.; PARK, J.H.; Ahn, Y.S.; Chung, K.C. Induction of Neuronal Cell Death by Rab5A-dependent Endocytosis of Alpha-synuclein. J Biol Chem Apr 20, 2001.

Tavares, A. - Doença de Alzheimer e as Demências do Idoso. Folha Med 5: 259-64, 1985

Tavares, A. - Demências. In: Pedrosa, E.R.P.; Rocha, M.O.C.; Silva: Medicina Geral de Adultos. Ed. Atheneu. São Paulo, pp. 436-44, 1992.

Togo, T.; Iseki, E.; MaruI, W.; AKIYAma, H.; UedA, K.; KosaKa, K. - Glial Involvement in the Degeneration Process of Lewy Body - Bearing Neurons and the Degradation Process of Lewy Bodies in Brains of Dementia with Lewy Bodies. J Neurol Sci 184(1): 71-5, 2001.

Tomlinson, B.E.; Blessed, G; Roth, M. - Observations on the brains of Demented old People. J Neurol Sci 11: 205-42, 1970.

Trojanowaski, JQ.; LeE, VMY. - Aggregation of Neurofilament and $\alpha$ Synuclein Proteins in Lewy Bodies. Arc Neurol 55: 151-2, 1998.

Tumbull, S.; Tabner, B.J.; El-Agnaf, O.M.; Moore, S.; Davies, Y.; Allsop, U.K. - Alpha-Synuclein Implicated in Parkinson's Disease Catalyses the Formation of Hydrogen Peroxide in Vitro. Free Radic Biol Med 15(30) (10): 1163-70, 2001.

Wesnes, K.A.; McKeith, I.G.; Ferrara, R.; Emre, M. et al. - Effects of Rivastigmine on Cognitive Function in Dementia with Lewy Bodies: a Randomised Placebo-Controlled International Study Using the Cognitive Drug Research Computerised Assessment System. Dement Geriatr Cogn Disord 13(3): 183-92, 2002.

Woulfe, J.; HoOgendoorn, H.; TARNopolsKy, M.; MunOZ, D.G - Monoclonal Antibodies Against Epstein-Barr Virus Cross-React with AlphaSynuclein in Human Brain. Neurology 55(9): 1398-401, 2000.

Zhang, J.; Perry, G; Robertson, D. et al. - Parkinson's Disease is Associated with Oxidative Damage to Cytoplasmic DNA and RNA in Substantia Nigra Neurons. Am J Pathol 154 (5): 1423-9, 1999. 